\title{
An Efficient Delivery System to Control and Eradicate Ebola
}

\author{
Dedi Li \\ School of Energy and Power Engineering, North China Electric Power University, Baoding 071000, \\ China \\ 836793581@qq.com
}

Keywords: System Classification; Equivalent Distance; AHP and Entropy method.

\begin{abstract}
In view of the delivery and distribution of emergency supplies such as vaccine and medication for disaster area of Ebola, an efficient delivery system has been proposed in this paper. Taking into account that there are only several country such as the U.S., Canada, Britain et al. can produce vaccine and drug for Ebola, the delivery system is divided into three level: world level, country level and city level. Then, the delivery rules of each level and model theory has been illustrate. By developing an equivalent distance model, which integrate the influence of city and disease factors, the distribution center in country level is determined. For an accurate result, Analytic Hierarchy Process method and Entropy method are employed to calculate weights of the equivalent distance.
\end{abstract}

\section{Introduction}

The West Africa, the most severely affected area by Ebola, have very weak health systems, lacking human and infrastructural resources, namely, to control and eradicate Ebola need supplies and resources from all over the world. Considering that there are scarce medicine and vaccine for the disease, an efficient delivery system should be build and establish realistic, sensible and useful rules to control and eradicate the spread of Ebola. In the paper, we develop the delivery system and analyze the distribution rules by simulation.

\section{Model for Delivery System}

\subsection{Model theory}

From website, we find that there are only several country such as the U.S., Canada, Britain et al. can produce vaccine and drug for Ebola. It means the vaccine and drug should be delivered by air transport to West Africa, where Ebola spread and outbreaks. Due to the international airport is scarce and stationary in countries of West Africa, the delivery system from producing countries to Ebola spread countries is simple and has less space to improvement. Therefore, we mainly discuss the delivery system in country. The rules of the delivery is as following:

- In the world level, the vaccine and drug of Ebola will be delivered by air transport from producing countries to the international airport in affected countries, the number of medicine and time to deliver is determined by every countries demand, namely the total cases of every country.

- In the country level, find a distribution center by calculating the equivalent distance of every point (city or area) and deliver the supplies to the distribution center from international airport.

- According to the number of infectious people and the spread process in each city or area, we determine the number of medicine and the time to deliver from distribution center.

- In city level, the delivery system is same as the country level, but the target point may be villages or small town 
Table 1 Symbols and Definitions

\begin{tabular}{cc}
\hline Symbols & Definitions \\
\hline$L_{s}$ & Sum of all equivalent distance from other city to $s^{\text {th }}$ city. \\
$l_{i}$ & Equivalent distance from $s^{\text {th }}$ city to $i^{\text {th }}$ city \\
$s_{i}$ & Real distance from $s^{\text {th }}$ to $i^{\text {th }}$ city \\
$\alpha_{i}$ & Equivalent factor of $i^{\text {th }}$ city \\
\hline
\end{tabular}

\subsection{Model Building}

We build the model for three aspects: the number of medicine demand, locations of delivery, and the time of delivery.

The quantity of medicine. In order to simply the model to obtain a useful result, we define the rules as follow:

- The world level's demand determined by the number of infectious people in every affected country.

- The country level's demand determined by the number of infectious people in every cities in the country.

- The city level's demand determined by the number of infectious people in every village or small town in the city.

The number of infectious people could be obtained by report of various area.

Locations of delivery. Due to the delivery system from world level to the country level is single, which is from producing countries to the international airport in affected country and the city level system is the same as country level, we determine to take country level as a case to build model.

In order to deliver the supplies in time, we calculate the equivalent distance of every city to find a distribution center, of which $L$ is the minimum compared with the other city.

- The equivalent distance

We define the equivalent distance from distribution center to $i^{\text {th }}$ city as follow:

$$
l=\alpha_{i} s_{i}
$$

- The equivalent factor $\alpha_{i}$

The equivalent factor integrate three factors of city, which can influence distance from the distribution center to the city.

- Spread trend of Ebola: Through the Spread Model above, we can obtain the spread trend of Ebola. if the spread trend is increase rapidly, the distance should be shorter.

- City size: A big city has better medical conditions, storage conditions and the traffic conditions, thus, if the city is a big one, it has more probability to be choose as the distribution center.

- City importance: Port and capital city should has a short distance from distribution center because of the higher contact rate of people.

Then the equivalent factor calculate as following:

$$
\alpha_{i}=\omega_{1} x_{i 1}+\omega_{2} x_{i 2}+\omega_{3} x_{i 3}
$$

Where $x_{i 1}, x_{i 2}, x_{i 3}$ is the three factors of $i^{\text {th }}$ city above?

- The weight $\omega_{1}, \omega_{2}, \omega_{3}$

In order to obtain the accurate weight of each aspect, we calculate every weight from subjective and objective aspects [1] and define the algorithm as following:

$$
\begin{gathered}
t_{i}=\sqrt{\left(v_{i} \bullet w_{i}\right)}, i=1,2, \ldots . . Q \\
\omega_{i}=t_{i} / \sum_{n=1}^{Q} t_{n}, i=1,2, \ldots ., Q
\end{gathered}
$$


Where $\omega_{i} v_{i} w_{i}$ is the $i^{\text {th }}$ property's combination weights, objective weights and subjective weights, $t_{i}$ is intermediate variable.

- Objective weight

We employ the Entropy Law to calculate the objective aspect as following:

1. Determine the normalized matrix

$$
\begin{gathered}
H=\left\{h_{i j}\right\}_{J \times Q} \\
h_{i j}=r_{i j} / \sum_{i=1}^{J} r_{i j}, i=1,2 \ldots J ; j=1,2 \ldots Q
\end{gathered}
$$

Where $i=1,2 \ldots J$ the number of is object and $j=1,2 \ldots Q$ is the number of index.

2. Calculate entropy of index $x_{i}$

$$
\begin{aligned}
& E_{i}=-\frac{1}{\ln J} \sum_{i=1}^{J}\left(h_{i j} \bullet \ln h_{i j}\right), j=1,2 \ldots Q \\
& \text { While } h_{i j}=0 \text {, define } h_{i j} \bullet \ln h_{i j}=0 .
\end{aligned}
$$

3. Calculate objective weight

$$
v_{i}=\left(1-E_{j}\right) / \sum_{K=1}^{Q}\left(1-E_{k}\right), j=1,2 \ldots Q
$$

- Subjective weight

We employ the AHP method to calculate the subjective aspect as following:

1. Determine the judging matrix

We use the pairwise comparison method and one-nine method to construct judging matrix $\mathrm{A}=\left(a_{i j}\right)$.

$$
a_{i j}=\frac{1}{a_{j i}}
$$

Where $a_{i j}$ is set according to the one-nine method.

2. Calculate the eigenvalues and eigenvectors

The greatest eigenvalue of matrix $\mathrm{A}$ is $\lambda_{\max }$, and the corresponding eigenvector is $u=\left(u_{1}, u_{2}, u_{3}, \ldots u_{n}\right)^{T}$. Then we normalize the $\mathrm{u}$ by the expression:

$$
x_{i}=\frac{u_{i}}{\sum_{i=0}^{n} u_{j}}
$$

3. Do the consistency check

The indicator of consistency check formula:

$$
C I=\frac{\lambda_{\max }-n}{n-1}
$$

Where $\mathrm{n}$ denotes the exponent number of matrix.

The expression of consistency ratio:

$C R=\frac{C I}{R I}$

- Determine the distribution center

The sum of all equivalent distance is

$L_{s}=l_{1}+l_{2}+\ldots . l_{J}$,

If the value of $L_{s}$ appears to be minimum, we can determine the $s^{\text {th }}$ city as the distribution center. 


\section{Sierra Lone Delivery System}

Take Sierra Lone as a case to operate our delivery model and number each city in Sierra Lone as the distribution center candidate. We can obtain the results as following.

Collect data (city population ${ }^{[2]}$, city cases ${ }^{[3]}$, distance between two city ${ }^{[4]}$, weather the capital or port ${ }^{[2]}$ ) of each city from website and carry out the equivalent distance model.

The top 3 city is as follow:

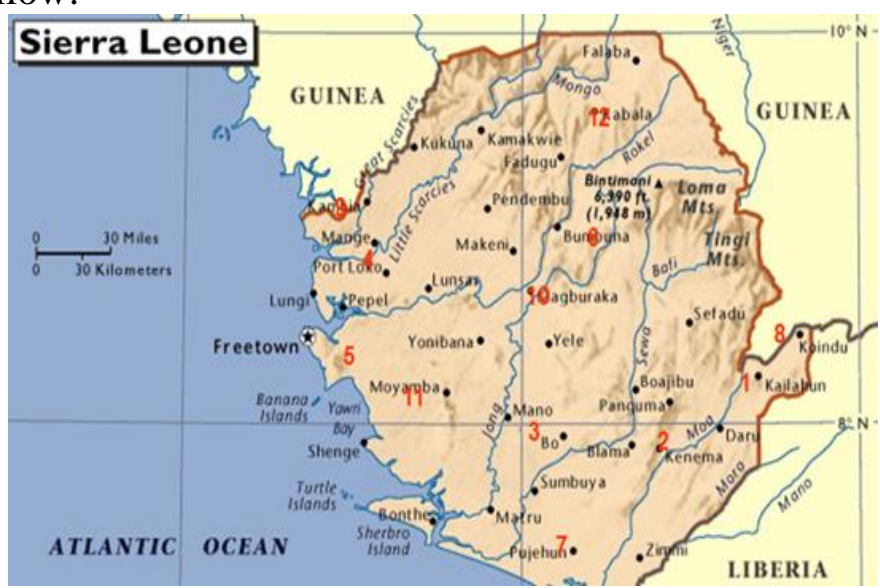

Figure 1 City number and distribution in Sierra Lone

Table 2 the top 3 city to be distribution center

\begin{tabular}{|c|c|c|}
\hline City Number & City Name & $L(\mathrm{~km})$ \\
\hline 11 & Moyamba & 553.5 \\
\hline 4 & Port Loko & 595.4 \\
\hline 3 & Bo & 615.9 \\
\hline
\end{tabular}

\section{Summary}

In this paper, an efficient and realistic delivery system has been proposed to control and eradicate Ebola in Western Africa. By dividing the system into three level and establish delivery rules, supplies and resources can be distributed reasonable in time. An equivalent distance model which integrate the city and disease factors is developed to determine the distribution center in country. Analytic Hierarchy Process method and Entropy method bring a more accurate result. Finally, take Sierra Lone as a case to build the delivery system and the city Moyamba is the best one to be distribution center.

\section{References}

[1] Yu Long Liang, Chen Jing Rong, Cui Xiao Chuan "A Traffic Network Route Choosing Method based on Combined Weight" Journal of Luoyang Institute of Science and Technology (Natural Science Edition):2014

[2] http://en.wikipedia.org/wiki/Sierra_Leone

[3] http://www.mofcom.gov.cn/article/i/jyjl/k/201502/20150200890064.shtml

[4] http://www.google.cn/maps 